\title{
Renal Transplant Pathology Series-II
}

\author{
Dr. Muhammed Mubarak \\ Professor of Pathology and HOD \\ JIK Department of Histopathology, \\ Sindh Institute of Urology and Transplantation (SIUT), \\ Karachi, Pakistan. \\ Email address: drmubaraksiut@yahoo.com
}

Pak J Kidney Dis 2020;4(2):257-259

Banff classification of renal allograft pathology represents an international consensus-based classification for the standardized reporting of renal allograft pathological lesions in both clinical practice and international clinical trials. This classification has evolved markedly over the past 30 years and has become a multidisciplinary undertaking. It has also increased in complexity and some categories have expanded substantially. In this pictorial, we aim to provide an illustrated presentation of the Banff classification categories and practical points to identify and report the lesions. This will be useful for better understanding of the renal transplant pathology not only for trainees and residents of nephrology and histopathology but also practicing pathologists and nephrologists.

\section{Banff category 1. Normal biopsy or nonspecific changes}

This category retains the top position among the six diagnostic categories of the Banff classification till date. This apparently seems to be a redundant category but the rationale behind retaining this appears to be the inadvertent consequence of over-zealous biopsy approach. In some centers, biopsies are done too quickly and for transient rises in serum creatinine, where the morphological lesions have not fully expressed, and biopsy appears normal. There can be genuine reasons for a normal appearance of renal allograft biopsy. Sometimes, systemic viral infections, such as CMV, may result in rise in serum creatinine but the renal allograft biopsy does not show viral inclusions or inflammation. In some cases, sampling error may account for the normal appearances of the sampled cores, especially when the rise in creatinine is significant. The prevalence of this finding in renal allograft biopsies should ideally be low. However, it partly depends on how quickly the biopsies are done, or how extensive pre-biopsy work-up for graft dysfunction is carried out before undertaking biopsy.

The definition of this category is self-explanatory and necessitates that all the four components of the kidney graft are morphologically normal or show minor nonspecific changes. In fact, this category is a diagnosis of exclusion, which means that not only the graft parenchyma should be free of infiltrate, but it should also show no acute tubular injury (ATI) or acute tubular necrosis (ATN). Hence, diagnosis of this category requires that the entire biopsy material be examined diligently and thoroughly, with multiple serial sections and sometimes sectioning of the whole biopsy material, especially if there is significant unexplained renal graft dysfunction. This is the only category of Banff classification which cannot occur concurrently with any other category, as alluded to earlier and is a diagnosis of exclusion. A series of representative images of the biopsy with normal category are illustrated in Figures 1 to 6 . The entire biopsy showed such changes and hence was diagnosed as normal biopsy.

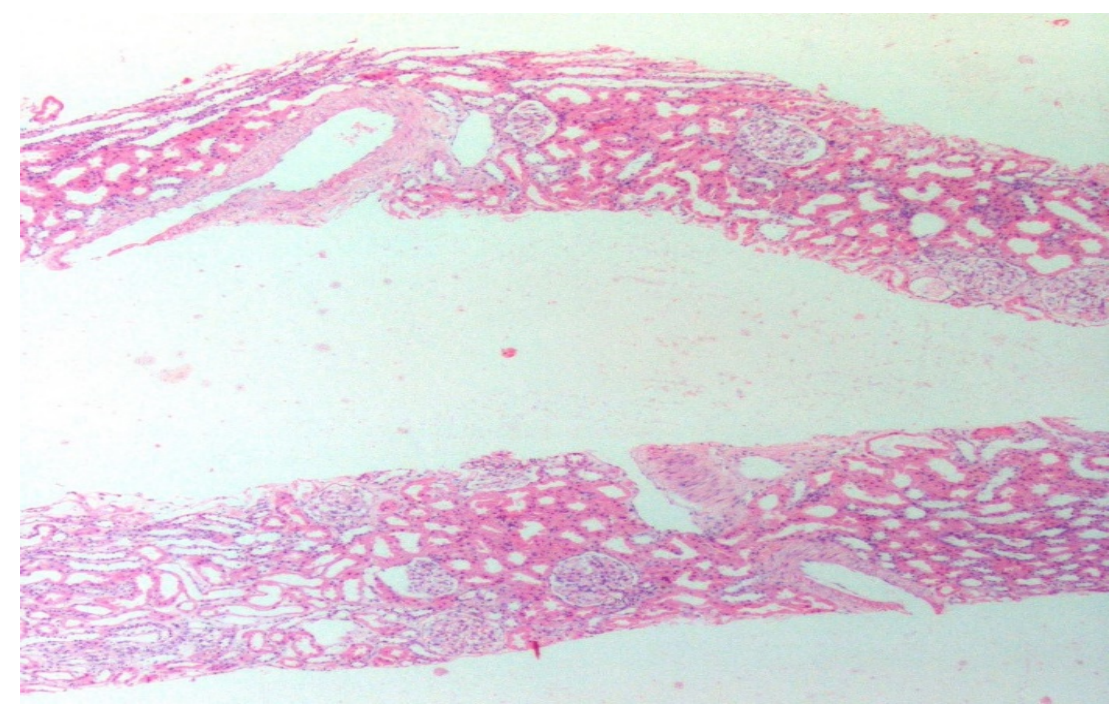

Figure 1. Low-power photomicrograph showing two cores of renal graft biopsy, both containing cortex in this area and displaying three components of the graft parenchyma: glomeruli, arteries or vessels and tubules. Interstitium is normally not visible under the light microscopy, as in the native kidney. It represents the main battlefield for $\mathrm{T}$ cellmediated rejection but there is no infiltrate in this biopsy and no significant acute tubular injury. (H\&E, $\times 50)$. 



Figure 3. Low-power photomicrograph from the same biopsy showing notable absence of chronic changes in the biopsy. All three components of the graft parenchyma are visible and appear unremarkable, even at this magnification. There is no interstitial infiltrate in the biopsy. (JMS, $\times 50$ ).
Figure 2. Medium-power photomicrograph of the same renal graft biopsy showing three components of the graft tissue in more detail. One interlobular size artery sits in the middle of the field. There is no arteritis, no glomerulitis, and no infiltrate in the biopsy to suggest rejection or infection and no significant acute tubular injury. (H\&E, x100).

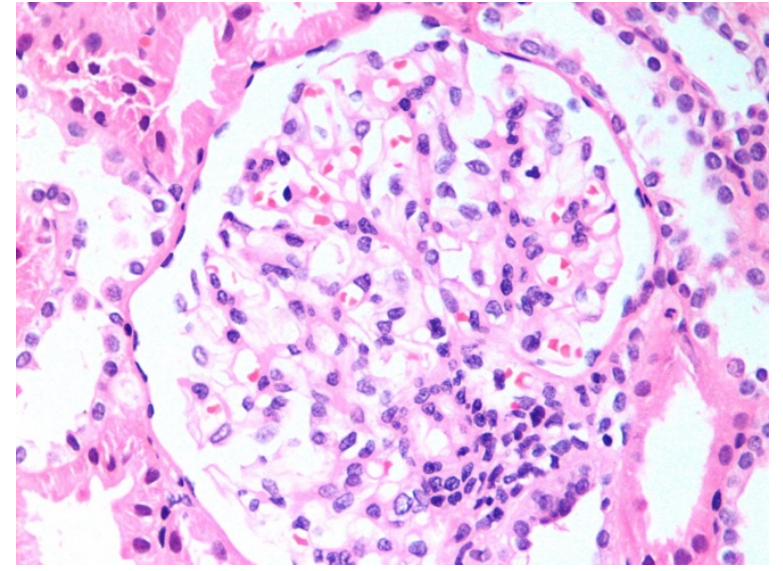

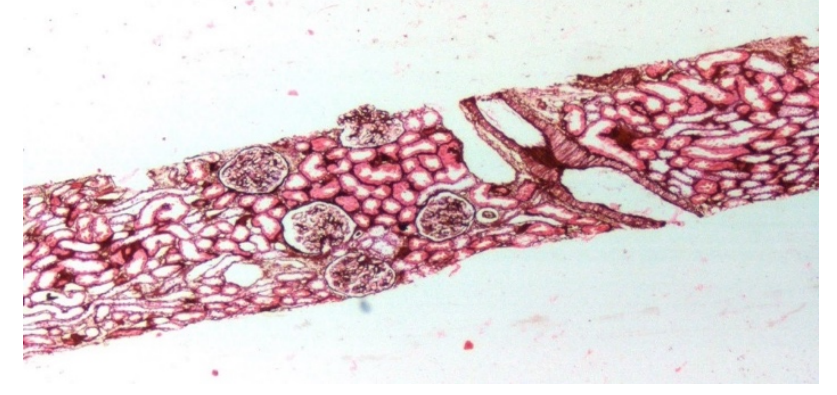

Figure 4. This is high-power photomicrograph of a representative glomerulus in the above graft biopsy. There is no glomerulitis. All capillary lumens are patent. There is no capillary occlusion and no endothelial cell swelling or inflammatory cell infiltration. No intralumenal thrombosis is seen in glomerular capillaries. There is no inflammatory cell infiltrate in the interstitium (H\&E, x400). 

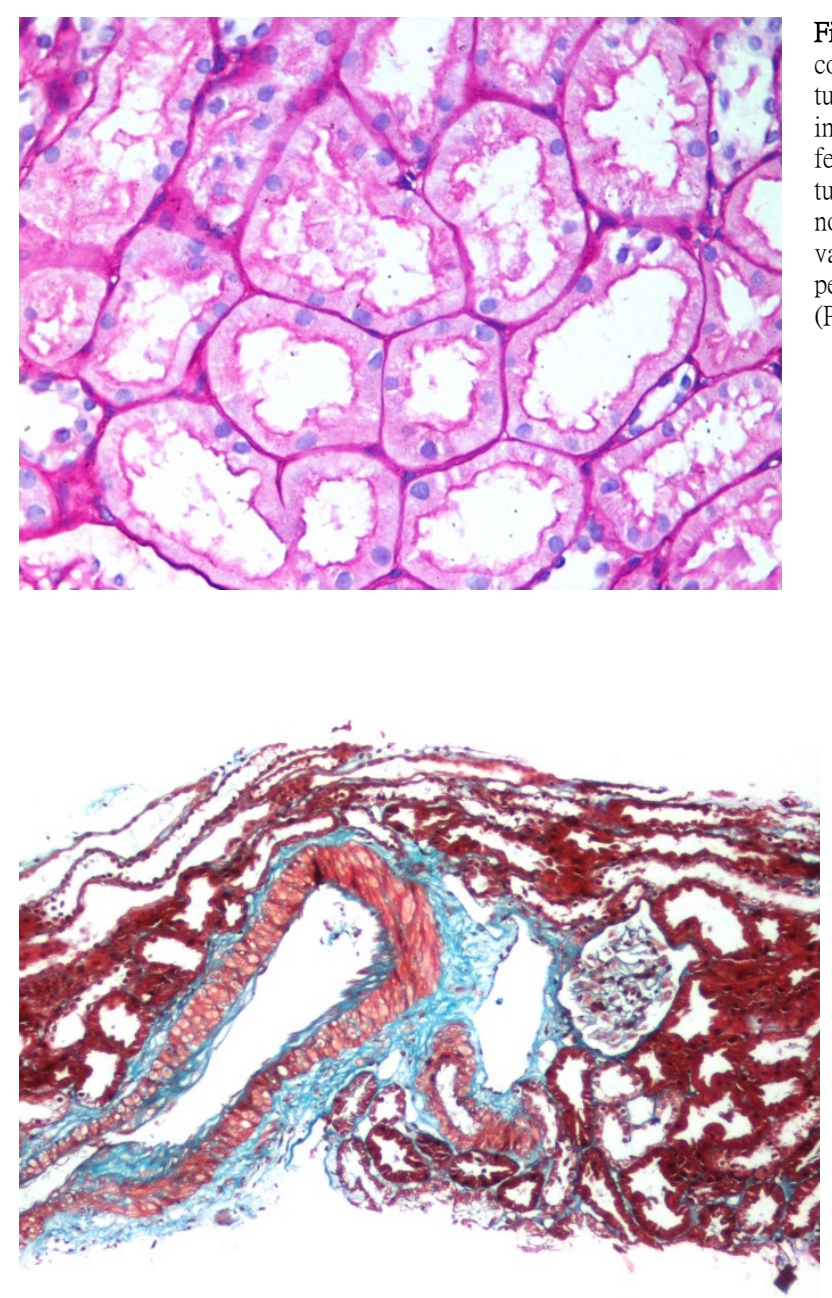

Figure 5. This is a high-power photomicrograph of the representative tubular component of the above biopsy. Majority of tubules in this field are proximal tubules. These are back to back in arrangement with no intervening interstitium visible. The lumina are also not patulous or widely dilated, a feature of acute tubular necrosis. The brush border is intact in most of the tubules. Hence, the height of the tubular epithelial cells is maintained. There is not much debris in the lumen or any dystrophic calcification, nor vacuolization of tubular epithelial cells to indicate acute tubular injury. No peritubular capillary dilatation is seen, nor is there any peritubular capillaritis (PAS, $\times 400)$.
Figure 6. This is medium-power photomicrograph of the biopsy all three components with an interlobular artery at the center of the field. This is a cross-section of the artery and a small vein surrounded by periadventitial fibrous tissue staining green with this special stain. This amount of fibrous tissue is normal surrounding larger vessels. There is no intimal arteritis, no transmural arteritis or fibrinoid necrosis. The lumen is also empty and no thrombus or extraneous material is seen. (Trichrome stain, $\times 100$ ).

\section{Further reading:}

1. Solez K. History of the Banff classification of allograft pathology as it approaches its 20th year. Curr Opin Organ Transplant 2010;15:49-51.

2. Solez K, Racusen LC. The Banff classification revisited. Kidney Int 2013;83:201-6.

3. Mubarak M, Kazi JI. Evolution of the Banff working classification of renal allograft pathology: updates and future directions. J Transplant Technol Res 2013;3:2.

4. Weening JJ. The art of classifying renal allograft pathology. Nat Clin Pract Nephrol 2008;4:420-1.

5. Roufosse C, Simmonds N, Clahsen-van Groningen M, et al. A 2018 Reference Guide to the Banff Classification of Renal Allograft Pathology [published correction appears in Transplantation. 2018 Dec;102(12):e497].Transplantation 2018;102:1795-1814. 